\title{
Validity and safety of endoscopic biliary stenting for biliary stricture associated with IgG4-related pancreatobiliary disease during steroid therapy
}

\section{(ㄷ)(우우}

\author{
Authors \\ Tetsuya Ito ${ }^{1}$, Hideaki Hamano ${ }^{1}$, Shigeyuki Kawa ${ }^{3}$ \\ Institutions \\ 1 Department of Gastroenterology, Shinshu University \\ School of Medicine, Matsumoto, Nagano, Japan \\ 2 Department of Gastroenterology, North Alps Medical \\ Center Azumi Hospital, Ikeda, Nagano, Japan \\ 3 Department of Internal Medicine, Matsumoto Dental \\ University, Shiojiri, Nagano, Japan
}

Yasuhiro Kuraishi ${ }^{1}$, Takashi Muraki ${ }^{2}$, Norihiro Ashihara', Makiko Ozawa ${ }^{1}$, Akira Nakamura ${ }^{1}$, Takayuki Watanabe ${ }^{1}$,

submitted 21.2.2019

accepted after revision 22.5.2019

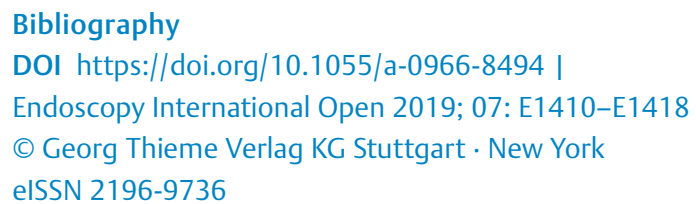

\section{Corresponding author}

Takashi Muraki, MD, PhD, Department of Gastroenterology, North Alps Medical Center Azumi Hospital, 3207-1 Ikeda, Ikeda Kitaazumi-gun, Nagano 399-8695, Japan

Fax: +81-261-622711

murakitakashi1108@gmail.com

\section{ABSTRACT}

Background Patients with IgG4-related sclerosing cholangitis and autoimmune pancreatitis frequently develop obstructive jaundice, which requires endoscopic biliary stenting (EBS) during steroid therapy to prevent bile duct infec- tion from cholestasis and adverse steroid effects. However, it is controversial whether EBS during steroid therapy is advisable, because the procedure itself carries a risk of cholangitis and procedure-related adverse events. This study aimed to clarify the validity and safety of EBS for patients with biliary stricture associated with IgG4-related pancreatobiliary disease (IgG4-PBD) during steroid therapy.

Methods We enrolled 59 patients who presented with biliary stricture exhibiting jaundice or liver dysfunction and who were treated with EBS. The incidences of recurrent biliary obstruction and acute cholangitis were compared for EBS cases with and without steroid administration.

Results EBS was present in 55 periods with steroid administration and 110 periods without. The incidence of recurrent biliary obstruction was significantly lower in cases with steroids than in those without (1-month no obstruction rate: $100 \%$ vs. $82 \%$; log-rank test $P=0.0015)$. The incidence of acute cholangitis related to stenting was significantly lower in cases with steroids than in those without (1-month no acute cholangitis rate: $100 \%$ vs. $90 \%$; logrank test $P=0.0278$ ). Biliary stents could be removed without acute cholangitis, liver dysfunction, or stent replacement in $96 \%$ of patients who underwent endoscopic retrograde cholangiopancreatography 1 month after commencing steroid administration.

Conclusions EBS during steroid administration was both valid and safe in patients with biliary stricture associated with IgG4-PBD. Stents could be safely removed 1 month after steroid initiation.

\section{Introduction}

IgG4-related sclerosing cholangitis (IgG4-SC) is a specific type of sclerosing cholangitis exhibiting elevation of serum IgG4 levels [1] and dense lymphoplasmacytic infiltration with extensive fibrosis in the bile duct wall [2]. Most cases of IgG4-SC coexist with type 1 autoimmune pancreatitis (AIP), both of which showing a favorable response to steroid therapy [3 - 5]. According to the cholangiographic classification of IgG4-SC [6], most patients are classified into type- $1 \mathrm{lgG} 4-\mathrm{SC}$ that shows stricture of the lower bile duct. However, it is controversial whether distal biliary strictures are true IgG4-SC or secondary biliary strictures compressed by the swollen pancreatic head in AIP. Therefore, this study collectively described biliary strictures related to IgG4-SC or AIP as biliary stricture associated with IgG4-related pancreatobiliary disease (IgG4-PBD), which included lower biliary strictures and strictures in other parts of the biliary system. On cholangiography of biliary strictures associated with 
IgG4-PBD, multifocal strictures of the intra- or extrahepatic bile ducts can resemble cholangiocarcinoma, pancreatic carcinoma, or primary sclerosing cholangitis (PSC). Thus, distinguishing the biliary stricture from those progressive diseases can be challenging during diagnosis [7-9]. Several reports have shown that endoscopic retrograde cholangiopancreatography (ERCP) [10-12] and its related procedures, such as intraductal ultrasonography (IDUS) [13-16] and transpapillary biliary biopsy $[3,16,17]$, are useful and very important tools for proper differentiation. As misdiagnosis and treatment should be avoided in malignant diseases, there are numerous advantages of ERCP and its related procedures for accurate diagnosis.

Patients with IgG4-PBD (IgG4-SC and AIP) frequently present with obstructive jaundice, the majority of whom receive endoscopic biliary stenting (EBS) for biliary drainage. Obstructive jaundice is found in $35-77 \%$ of patients presenting with IgG4SC $[3,18,19]$. Globally, most patients $(71 \%)$ with AIP and jaundice undergo EBS [20]. The primary aim of EBS is to prevent acute cholangitis, which is a serious adverse event leading to septicemia. It has been reported that $10 \%$ of patients with IgG4-SC develop acute cholangitis at presentation [19]. Acute cholangitis also occurs as an ERCP-related adverse event in $0.5 \%$ to $3 \%$ of cases [21-26]. Jaundice and incomplete biliary drainage have been reported as significant risk factors for post-ERCP acute cholangitis [25, 27], with the latter representing the strongest influence on cholangitis risk [28]. Thus, EBS is necessary for IgG4-PBD with jaundice to prevent acute postERCP complications.

It has also been reported that obstructive jaundice in AIP can be treated with steroid therapy without EBS [29]. Improved liver tests within a few days after steroid therapy without apparent acute cholangitis indicated that ERCP could be reserved for cases restricted to intermediate imaging findings or unresponsiveness to steroid therapy. It may also be possible to treat IgG4-PBD with jaundice by steroid therapy without biliary stenting in selected clinical settings. Moreover, EBS during steroid administration carries the potential of inducing acute cholangitis due to drug-induced immune suppression.

Based on the above, it is controversial whether EBS during steroid administration is advisable for patients with biliary stricture associated with IgG4-PBD. Steroid therapy is the established treatment for IgG4-PBD. As there have been no direct comparisons of EBS in patients having biliary stricture associated with IgG4-PBD with and without steroid administration, this study sought to clarify the validity and safety of EBS for patients with steroid administration.

\section{Patients/materials and methods}

\section{Patients}

A total of 104 patients with biliary stricture associated with IgG4-PBD were treated between January 1996 and November 2017 at Shinshu University Hospital in Matsumoto, Japan. All patients were diagnosed with IgG4-SC according to the clinical diagnostic criteria established by the Japanese Biliary Association in 2012 [6]. Based on these criteria, patients with distal biliary stricture possibly associated with AIP were included in
Patients with biliary stricture associated with IgG4-related pancreato-biliary disease $(n=104)$

Diagnosis of IgG4-SC according to the clinical diagnostic criteria established by the Japanese Biliary Association (2012)

\section{Excluded}

- Normal serum biochemical liver tests $(n=41)$

EBS performed $(n=63)$

\section{Excluded}

- Associated with choledocholithiasis $(n=2)$

- Underwent surgery for preoperative diagnosis of malignant disease $(n=2)$

Included in this study $(n=59)$

- Fig. 1 Inclusion criteria for this study and flow diagram. A total of 104 patients with biliary stricture associated with IgG4-related pancreatobiliary disease were identified. Among them, 63 patients underwent endoscopic biliary stenting (EBS) and 59 patients were ultimately evaluated in this study.

IgG4-SC. All medical records were reviewed retrospectively. Among 63 patients who received EBS, four were excluded due to choledocholithiasis or surgery due to malignant disease. The remaining 59 patients were enrolled in this study ( Fig. 1). EBS was performed on the following patients having a risk of post-ERCP acute cholangitis before steroid therapy: (1) those with jaundice or serum elevation in biochemical liver tests, and (2) those with biliary strictures and marked dilatation of the proximal bile duct despite no jaundice at the time of ERCP but having a risk of jaundice soon after ERCP-related procedures. Steroid therapy was commenced after careful diagnosis. The initial oral prednisolone dose for remission induction was $0.6 \mathrm{mg} / \mathrm{kg} /$ day for approximately 4 weeks. The dose was then tapered by $5 \mathrm{mg}$ every $1-2$ weeks based on the Japanese consensus guidelines for AIP [30].

\section{ERCP-related procedures}

Patients were treated based on the following endoscopic strategy for the management of biliary stricture associated with IgG4-PBD. We first performed ERCP ( $>$ Fig. 2a, b) and its related procedures, including IDUS ( $\boldsymbol{F}$ Fig. $\mathbf{2 c}$ ) and transpapillary biliary biopsy ( $\triangleright$ Fig. $\mathbf{2 d}$ ), in one session for the diagnosis, especially to distinguish this condition from pancreatic head carcinoma, cholangiocarcinoma, and PSC. Rectal diclofenac $(25 \mathrm{mg}$ in patients $<50 \mathrm{~kg}$ or $50 \mathrm{mg}$ in patients $\geq 50 \mathrm{~kg}$ ) was administered for the prevention of post-ERCP pancreatitis just before the procedure. Antibiotics ( $2 \mathrm{~g}$ piperacillin) were also given prophylactically to prevent post-ERCP acute cholangitis. We evaluated adverse events at the time of ERCP, such as post-ERCP pancreatitis, bleeding, perforation, and post-ERCP acute cholangitis. The 

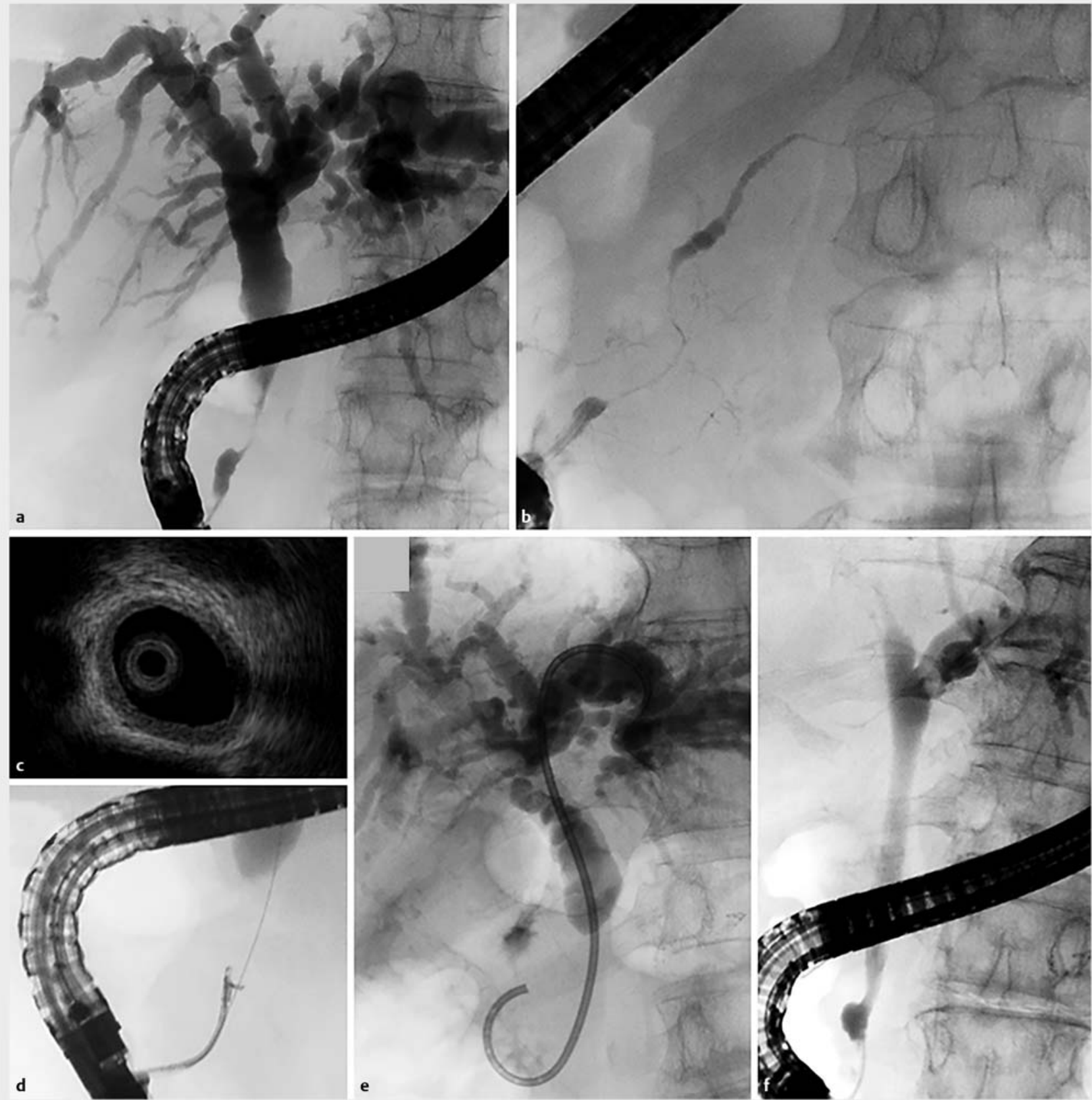

d

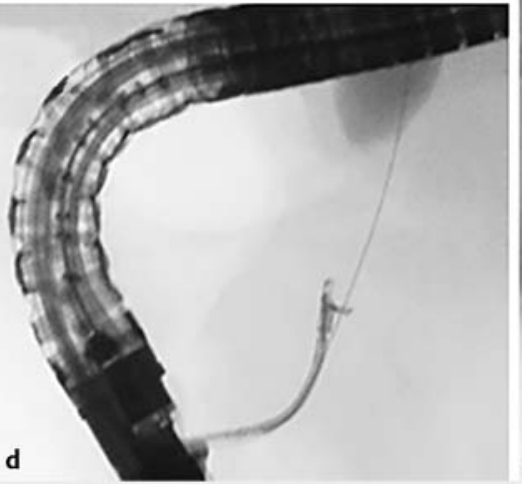

- Fig. 2 Endoscopic retrograde cholangiopancreatography (ERCP) and its related procedures in patients with biliary stricture associated with IgG4-related pancreatobiliary disease. a Cholangiography showed biliary strictures with a smooth surface and dilatation of the proximal bile duct that mimicked cholangiocarcinoma and pancreatic carcinoma of the head. Biliary stricture of the distal common bile duct was observed more frequently among patients with biliary stricture associated with IgG4-related pancreatobiliary disease. b Pancreatography showed diffuse irregular narrowing, a specific feature of autoimmune pancreatitis. c Intraductal ultrasonography disclosed a smooth inner margin and homogeneous internal echo in the stricture despite the bile duct appearing normal in the cholangiogram. $\mathbf{d}$ Transpapillary forceps biopsy was useful to distinguish from pancreatobiliary carcinoma. Abundant IgG4-positive cells were often observed. e A biliary stent (7-Fr double pig-tail plastic stent) was placed into the intrahepatic bile duct across the biliary stricture. $\mathbf{f}$ Cholangiography 1 month after starting steroid therapy revealed improvement in the biliary stricture such that the biliary stent could be removed in almost all patients. 


\section{Representative case 1}

ERCP for diagnosis

EBS
Steroid

administration

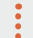

1 period of stenting

WITHOUT steroids

Representative case 2

Prior hospital

EBS

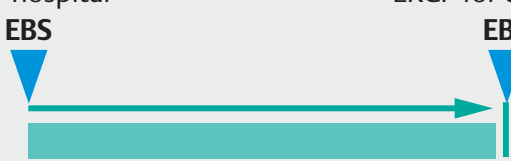

b
Stent removal

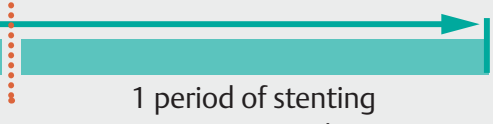

\section{WITH steroids}
1 period of stenting

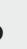
WITHOUT steroids
1 period of stenting
WITHOUT steroids

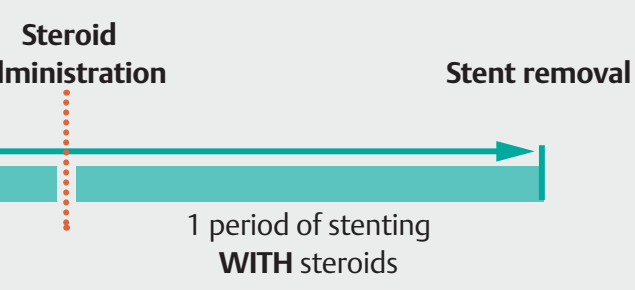

\section{Representative case 3}

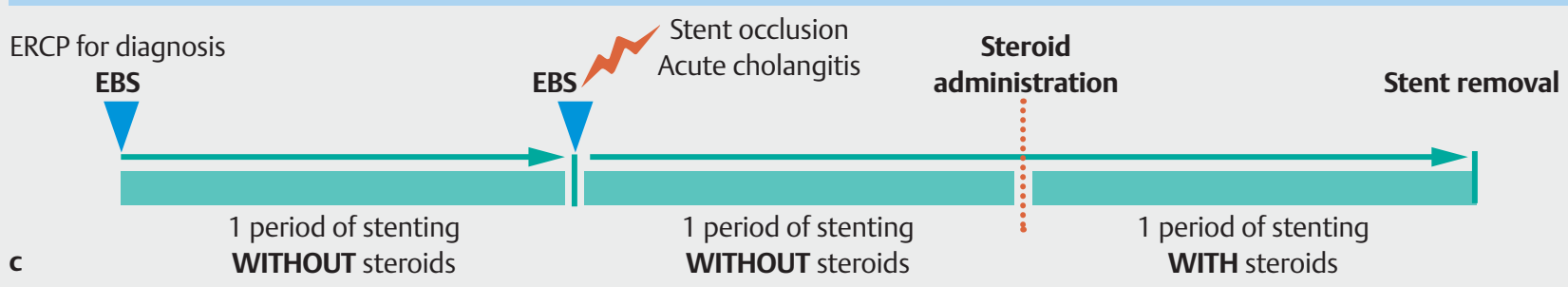

Fig. 3 Representative cases of endoscopic biliary stenting (EBS) with and without steroid therapy in this study. a A period of EBS without steroids was defined as the time from the insertion of a biliary stent to that of its removal or the commencement of steroids. An EBS period with steroids was defined as the time from the start of steroid administration to that of stent removal or from exchanging a stent to its removal during steroid therapy. For typical cases receiving steroid administration, treatment consisted of periods without steroids, after which the stent was not exchanged, and with steroids. b For cases receiving ERCP-related procedures, stent exchange was performed every time. $\mathbf{c}$ For cases of stent dysfunction, stent exchange was performed every time.

definition of ERCP-related adverse events was based on the criteria of Cotton et al. [31].

\section{EBS procedures}

During ERCP, stents were inserted along a guidewire under endoscopic and fluoroscopic guidance. A 7-Fr double pig-tail plastic stent (PBD-203-0707 or 07105, Olympus, Tokyo, Japan) was placed into the intrahepatic bile duct across the biliary stricture ( $\triangleright$ Fig. 2 e) to avoid stent deviation since the majority of biliary strictures when associated with IgG4-PBD improved after steroid administration. In patients with prior biliary stenting who were referred from other hospitals or who were suspected of a malignant disease, we performed ERCP again for accurate diagnosis and inserted a new biliary stent.

To estimate the effectiveness of steroid therapy for biliary stricture associated with IgG4-PBD, we routinely performed ERCP 1 month after the commencement of steroid remission induction therapy and removed the biliary stent upon confirmation of a smooth flow of contrast medium into and out of the stricture on cholangiography ( $\triangleright$ Fig. 2f). In patients with in- conclusive differentiation from malignant diseases, ERCP was carried out within 2 weeks after starting steroid remission induction therapy, and close follow-up was conducted for at least 1 year.

To evaluate the safety of EBS during steroid administration, we compared the incidence of recurrent biliary obstruction and acute cholangitis related to the stenting procedure between cases with and without steroid therapy. According to TOKYO criteria 2014 [32], recurrent biliary obstruction was defined as a composite end point of either occlusion or deviation/migration. A period of EBS without steroids was defined as the time from the insertion of a biliary stent to that of its removal or the commencement of steroids. An EBS period with steroids was defined as the time from the start of steroid administration to that of stent removal or from exchanging a stent to its removal during steroid therapy ( $\triangleright$ Fig.3). In typical cases of steroid administration, EBS treatment was divided into two periods: without steroids, after which the stent was not exchanged, and with steroids ( $\triangleright$ Fig. $3 a$ ). In patients who received ERCP-related procedures for more accurate diagnosis of IgG4- 


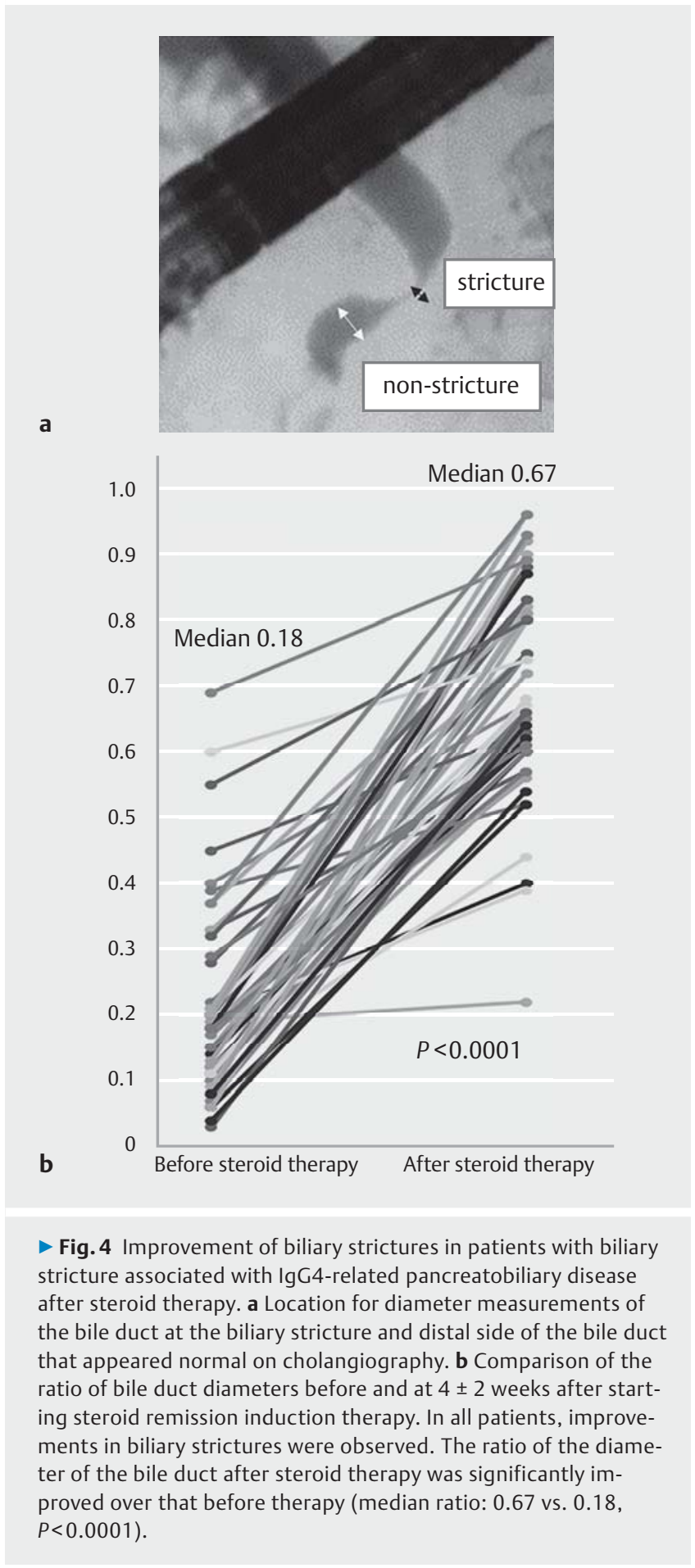

PBD or stent dysfunction, stent exchange was performed every time ( $\triangleright$ Fig. $\mathbf{3 b}, \mathbf{c}$ ).

To examine the improvement in biliary strictures, the diameter of the bile duct was measured at the biliary stricture and at the distal side of the bile duct that appeared normal on cholangiography ( $>$ Fig.4a). The ratio of these diameters was compared before and after steroid remission induction therapy. The patients were carefully monitored for evidence of biliary ad- verse events, including elevation of serum biochemical liver tests and acute cholangitis within 1 month after stent removal.

\section{EBS-related adverse events}

Stent-related adverse events including stent occlusion and deviation/migration were investigated in each period comparing patients with and without steroid administration. Stent occlusion was defined as abnormal serum values of liver function tests, the presence of symptoms due to biliary obstruction (abdominal pain/discomfort, nausea, and vomiting), or acute cholangitis with biliary dilatation on abdominal ultrasonography and/or computed tomography. Acute cholangitis was based on clinical evidence of infection (fever, leukocytosis, and abdominal pain) in patients with elevated serum biochemical liver tests. Biliary stent exchange was performed immediately in these patients.

\section{Statistical analysis}

For patient characteristics, continuous variables were expressed as median and range and categorical variables were expressed as number and percentage. Pearson's chi-squared test was adopted to test for differences between subgroups of patients. To compare continuous data, the Mann-Whitney $U$ test was employed. The time to recurrent biliary obstruction and acute cholangitis for stenting was calculated using the Kaplan-Meier method, and differences in rates with and without steroid administration were compared with the log-rank test. Comparisons of changes in the biliary diameter of strictures before and after steroid administration were performed using the Wilcoxon signed-rank test. A $P$ value of $<0.05$ was considered statistically significant. All statistical analyses were performed using JMP Statistics software version 13 (JMP, Tokyo, Japan).

\section{Ethics}

This study was performed in accordance with the current ethical guidelines of the Declaration of Helsinki and was conducted in accordance with the requirements of the Institutional Review Board of Shinshu University School of Medicine (approval number: 3913, approval date: 16 January 2018).

\section{Results}

Fifty-nine patients (42 male, median age: 66 years) received diagnostic ERCP and its related procedures followed by EBS for biliary drainage. Two patients exhibited acute cholangitis at presentation before the first ERCP. The baseline characteristics of the cohort are shown in $>$ Table 1. Type 1 AIP was observed in 57 patients (97\%). Biliary strictures limited to the intrapancreatic bile duct were seen in 48 patients ( $81 \%$ ) and strictures of hilar or intrahepatic bile ducts were found in 11 patients (19\%). Other organ involvements, including retroperitoneal fibrosis and enlarged salivary/lachrymal glands, were observed in 29 patients (49\%). Steroids were administered to 57 patients $(97 \%)$, with the remaining two patients refusing treatment. Serum IgG4 concentration was elevated $(>135 \mathrm{mg} / \mathrm{dL})$ in 43 of 52 patients (83\%) before therapy. 
- Table 1 Baseline characteristics of patients $(n=59)$ with biliary stricture associated with IgG4-related pancreatobiliary disease.

\begin{tabular}{|c|c|}
\hline \multicolumn{2}{|l|}{ Patient characteristic } \\
\hline - Gender (male), n (\%) & $42(72 \%)$ \\
\hline - Age, median [range], years & $66[47-92]$ \\
\hline - Complicated with type 1 AIP, n (\%) & $57(97 \%)$ \\
\hline - Hilar/intrahepatic biliary stricture, n (\%) & $11(19 \%)$ \\
\hline - OOI, n (\%) & $29(49 \%)$ \\
\hline - Steroid therapy, n (\%) & $57(97 \%)$ \\
\hline \multicolumn{2}{|c|}{ Serologic test before endoscopic biliary stenting, median [range] } \\
\hline - $\lg \mathrm{G}, \mathrm{mg} / \mathrm{dL}$ & $1850[666-3861]$ \\
\hline - $\operatorname{lgG} 4, \mathrm{mg} / \mathrm{dL}$ & $408[4-1660]$ \\
\hline " T-bil, mg/dL & $3.35[0.63-22.1]$ \\
\hline - $A L P, U / L$ & $1150[207-2938]$ \\
\hline - YGTP, U/L & $676[31-2642]$ \\
\hline
\end{tabular}

\section{Adverse events of ERCP and its related procedures}

At the time of ERCP for diagnosis, endoscopic sphincterotomy, IDUS, and transpapillary biliary biopsy were performed in 34 (58\%), 47 (80\%), and 47 (80\%) patients, respectively. Biliary biopsy was routinely performed to exclude malignant diseases. No patient received a histological diagnosis of malignancy. Two
- Table 2 Endoscopic retrograde cholangiopancreatography (ERCP)related procedures $(n=59)$ and their adverse events.

ERCP-related procedure, $\mathrm{n}(\%)$

- Endoscopic sphincterotomy

$34(58 \%)$

- Intraductal ultrasonography $47(80 \%)$

- Biliary biopsy $47(80 \%)$

ERCP-related adverse events, $n$ (\%)

- Post-ERCP pancreatitis $2(3 \%)$

- Immediate post-ERCP cholangitis

- Perforation 0

- Bleeding 0

ERCP, endoscopic retrograde cholangiopancreatography.

patients suffered from mild acute pancreatitis after ERCP that resolved within several days, whereas there were no early ERCP-related adverse events, such as perforation, bleeding, or post-ERCP acute cholangitis. Details of the procedures and their relatively few adverse events are summarized in $\triangleright$ Table 2 .

\section{Safety of EBS}

In the 59 patients, EBS was present in 55 periods with steroid administration and 110 periods without steroid administration. The baseline characteristics of the two groups were similar since they were composed of almost the same patients ( $\triangleright \mathrm{Ta}$ ble 3). One patient under steroid administration developed biliary bleeding 53 days after placement of a biliary stent, followed

- Table 3 Comparisons between baseline characteristics of patients receiving endoscopic biliary stenting (EBS) with and without steroid administration.

\begin{tabular}{|c|c|c|c|}
\hline & $\begin{array}{l}\text { EBS with steroid administration } \\
(n=55)\end{array}$ & $\begin{array}{l}\text { EBS without steroid administration } \\
(n=110)\end{array}$ & $P$ value \\
\hline Gender (male), n (\%) & $39(71 \%)$ & $72(66 \%)$ & 0.4815 \\
\hline Age, median [range], years & $66[47-92]$ & $67[47-92]$ & 0.8006 \\
\hline Type 1 AIP, n (\%) & $53(97 \%)$ & $108(98 \%)$ & 0.4741 \\
\hline Hilar/intrahepatic biliary stricture, n (\%) & $8(15 \%)$ & $24(22 \%)$ & 0.2654 \\
\hline OOI, n (\%) & $27(49 \%)$ & $48(44 \%)$ & 0.5071 \\
\hline Serum IgG, median [range], mg/dL & $1930[997-3861]$ & $1914[666-3861]$ & 0.9476 \\
\hline Serum IgG4, median [range] mg/dL & $404[4-1660]$ & $459[4-1660]$ & 0.5469 \\
\hline Serum T-bil, median [range], mg/dL & $3.65[0.6-22.1]$ & $2.6[0.6-22.1]$ & 0.2183 \\
\hline Serum ALP, median [range], U/L & $1151[245-2938]$ & $1283[207-2938]$ & 0.4784 \\
\hline Serum $\gamma G T P$, median [range], U/L & $671[116-2642]$ & $626[31-2642]$ & 0.7980 \\
\hline Ratio of biliary stricture ${ }^{1}$ & $0.19[0.04-0.69]$ & $0.19[0.03-0.69]$ & 0.6414 \\
\hline \multicolumn{4}{|c|}{$\begin{array}{l}\text { AIP, autoimmune pancreatitis; OOI, other organ involvements apart from type } 1 \text { AIP; IgG, immunoglobulin G; EBS, endoscopic biliary stenting; IgG4, immunoglo- } \\
\text { bulin G4; T-bil, total bilirubin; ALP, alkaline phosphatase; } \mathrm{YGTP} \text {, y glutamyl transferase. } \\
\text { 'Ratio of bile duct diameter between portions of biliary stricture and distal-side non-stricture on cholangiography. P values were calculated using the Mann-Whitney } \\
\text { U test and Pearson's chi-squared test. }\end{array}$} \\
\hline
\end{tabular}




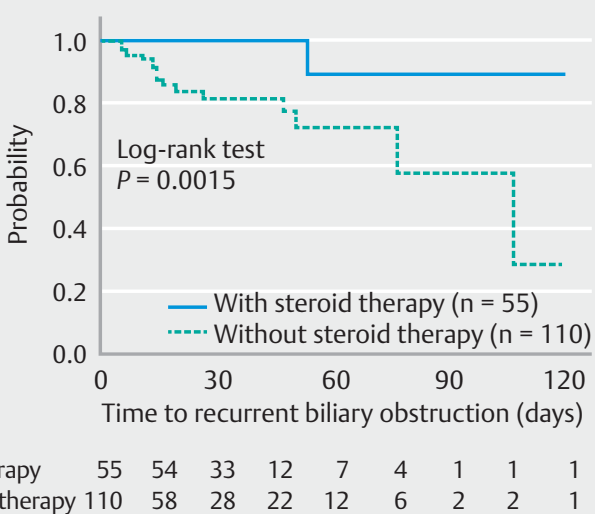

$\begin{array}{lrlllrllll}\text { With steroid therapy } & 55 & 54 & 33 & 12 & 7 & 4 & 1 & 1 & 1 \\ \text { Without steroid therapy } & 110 & 58 & 28 & 22 & 12 & 6 & 2 & 2 & 1\end{array}$ a

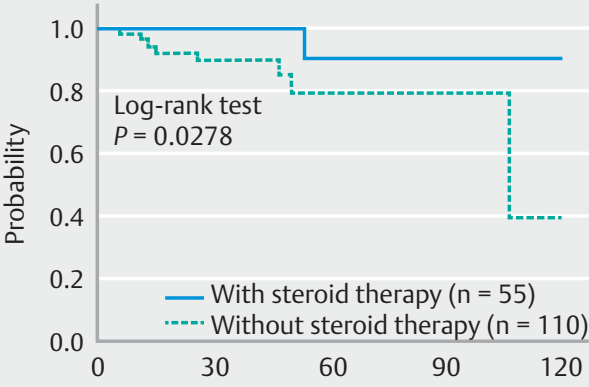

Patients at risk Time to acute cholangitis for stenting (days) $\begin{array}{llllllllll}\text { With steroid therapy } & 55 & 54 & 33 & 12 & 7 & 4 & 1 & 1 & 1\end{array}$ $\begin{array}{lllllllll}\text { Without steroid therapy } 110 & 58 & 28 & 22 & 12 & 6 & 2 & 2 & 1\end{array}$ b

- Fig. 5 Kaplan-Meier curves. a The time to recurrent biliary obstruction between stenting with and without steroid administration. The incidence of recurrent biliary obstruction with steroids was significantly lower than without (1-month no obstruction rate: $100 \%$ vs. $82 \%$; log-rank test $P=0.0015$ ). b The time to acute cholangitis for stenting. The incidence of acute cholangitis during stenting with steroids was significantly lower than without (1-month no acute cholangitis rate: $100 \%$ vs. $90 \%$; log-rank test $P=0.0278)$.

by acute cholangitis. In other patients, no episodes of acute cholangitis, stent occlusion, or stent migration were seen. Seven episodes (6\%) of stent occlusion and 10 episodes ( $9 \%$ ) of acute cholangitis were noted in the biliary stenting group without steroid administration. Stent tearing during removal was seen in one session. Although patients with prior biliary stenting at other hospitals before steroid therapy were included in this study, no statistically significant difference was seen for the time to recurrent biliary obstruction between EBS performed at our hospital and at others (log-rank test: $P=0.9654$ ).

The time to recurrent biliary obstruction was compared between groups with and without steroid administration. The incidence of recurrent biliary obstruction with steroids was statistically significantly lower than those without (log-rank test: $P=$ $0.0015)$. The cumulative rate of no recurrent biliary obstruction 1 month after biliary stenting with and without steroid administration was $100 \%$ and $82 \%$, respectively. The median time to recurrent biliary obstruction without steroid administration was 108 days ( $>$ Fig. 5a).
Next, the time to acute cholangitis for stenting was compared between groups with steroid administration and those without. The incidence of acute cholangitis during biliary stenting was significantly lower in cases receiving steroids (log-rank test $P=0.0278$ ). The cumulative rate of no acute cholangitis 1 month after biliary stenting with and without steroid administration was $100 \%$ and $90 \%$, respectively. The median time to acute cholangitis without steroid administration was 108 days ( $\vee$ Fig.5b).

\section{Timing of ERCP and stent removal after steroid remission induction therapy}

At ERCP performed 1 month after commencing steroid therapy as a standard strategy, we evaluated the effectiveness of steroids and the possibility of stent removal. In 45 patients, ERCP was performed 1 month \pm 2 weeks after the start of treatment. The extent of biliary stricture in cholangiography was compared before and after steroid therapy by calculating the ratio of bile duct diameters at the biliary stricture and at the non-stricture distal side ( $\mathbf{F i g . 4 a )}$. In all patients, an improvement in biliary strictures was found after steroid therapy with respect to diameter ratio ( $\vee \mathbf{F i g . 4 b}$ ), with the median diameter of biliary strictures being significantly improved by steroid therapy (0.67 vs. $0.18, P<0.0001)$. In 43 of 45 patients (96\%), biliary stents could be removed in a median period of 28 days following steroid commencement. After stent removal, no patients required re-stenting for biliary drainage or presented with acute cholangitis or elevation of serum biochemical liver tests.

\section{Discussion}

The present study revealed that the incidence of recurrent biliary obstruction and acute cholangitis after EBS for biliary stricture was significantly lower in IgG4-PBD patients with steroid administration than in those without. Biliary stents could be removed in $96 \%$ of patients who were free of acute cholangitis and liver dysfunction and did not require replacement. Recurrent biliary obstruction and acute cholangitis were also significantly lower in stenting groups with steroid administration, likely due to rapid pharmaceutical improvement in the biliary stricture. Iwasaki et al. [33] performed ERCP 1 - 4 weeks after steroid administration in 18 of 29 patients with IgG4-SC and found that lower bile duct strictures were improved in $100 \%$ of subjects and hilar and/or intrahepatic bile duct strictures were resolved in $75 \%$. In agreement with their report, the present study showed the amelioration of biliary strictures in all patients who underwent ERCP at 1 month after commencing steroid therapy. We considered that the dramatic and rapid effect of steroids on biliary strictures could prevent EBS-related adverse events, such as stent occlusion and cholangitis.

Although ERCP and its related procedures are useful for the diagnosis of IgG4-PBD, it must be stressed that these invasive procedures are sometimes associated with acute pancreatitis and other serious adverse events. However, Naitoh et al. [34] observed that the incidence of ERCP-related complications was lower in patients with AIP and IgG4-SC compared with non-AIP and non-IgG4-SC groups, and concluded that diagnos- 
tic ERCP and its related procedures were safe and acceptable for AIP and IgG4-SC patients. They also reported the incidence of pancreatitis to be $5.4 \%$ in the non-AIP cohort and $1.2 \%$ in the AIP cohort. Similarly, the present study uncovered few ERCPrelated adverse events (two cases of mild pancreatitis), supporting the view that these procedures were safe for biliary strictures associated with IgG4-PBD.

Following diagnostic ERCP for IgG4-PBD, it is recommended that EBS be performed afterwards to prevent acute post-ERCP cholangitis. Prompt steroid therapy is also advised after ERCP and EBS for biliary strictures associated with IgG4-PBD to improve the strictures and prevent EBS-related adverse events, such as acute cholangitis and stent occlusion.

We routinely performed ERCP 1 month after steroid commencement to assess the effectiveness of steroid therapy and evaluate the possibility of stent removal. Almost all stents could be removed at this point in our cohort, although some reports have shown that the optimal timing might be earlier. Iwasaki et al. [33] observed that all 10 AIP patients with jaundice could successfully undergo biliary stent removal after a mean interval of 16 days (range: 9 - 30 days). Moon et al. [35] reported that all 15 AIP patients had responded to steroid therapy in a 2-week trial, which resulted in an improvement in main pancreatic duct narrowing and a reduction in pancreatic mass. Thus, it may be possible to evaluate the effectiveness of steroid therapy earlier and remove stents as a result of the dramatic and rapid response to steroids. Prospective studies are required to determine the most appropriate timing for evaluating steroid therapy and stent removal.

This study had several limitations. First, the extent of jaundice and liver dysfunction at EBS varied because the timing of the technique was according to each physician's discretion. Several patients without jaundice were also included in the cohort. Second, the time to recurrent biliary obstruction in patients with steroid administration may not have been completely accurate since stents were not exchanged exactly at the start of steroid administration. However, this limitation could have resulted in a longer time to recurrent biliary obstruction in the stenting with steroid group, and so it was not necessarily a negative limitation of this study.

In conclusion, EBS during steroid administration is both valid and safe in patients with biliary stricture associated with IgG4PBD, with stents being removed safely 1 month after steroid remission induction therapy. Larger prospective multi-institutional studies are warranted to confirm our results and ascertain the optimal time of stent removal.

\section{Competing interests}

None

\section{References}

[1] Hamano H, Kawa S, Horiuchi A et al. High serum IgG4 concentrations in patients with sclerosing pancreatitis. NEJM 2001; 344: $732-738$

[2] Zen Y, Harada K, Sasaki M et al. IgG4-related sclerosing cholangitis with and without hepatic inflammatory pseudotumor, and sclerosing pancreatitis-associated sclerosing cholangitis: do they belong to a spectrum of sclerosing pancreatitis? Am J Surg Pathol 2004; 28 : $1193-1203$

[3] Ghazale A, Chari ST, Zhang L et al. Immunoglobulin G4-associated cholangitis: clinical profile and response to therapy. Gastroenterology 2008; 134: $706-715$

[4] Hirano K, Tada M, Isayama $\mathrm{H}$ et al. Long-term prognosis of autoimmune pancreatitis with and without corticosteroid treatment. Gut 2007; 56: $1719-1724$

[5] Nakazawa T, Ohara H, Sano $\mathrm{H}$ et al. Clinical differences between primary sclerosing cholangitis and sclerosing cholangitis with autoimmune pancreatitis. Pancreas 2005; 30: 20-25

[6] Ohara H, Okazaki K, Tsubouchi H et al. Clinical diagnostic criteria of IgG4-related sclerosing cholangitis 2012. J Hepatobiliary Pancreat Sci 2012; 19 : $536-542$

[7] Hamano H, Kawa S, Uehara T et al. Immunoglobulin G4-related lymphoplasmacytic sclerosing cholangitis that mimics infiltrating hilar cholangiocarcinoma: part of a spectrum of autoimmune pancreatitis? Gastrointest Endosc 2005; 62: $152-157$

[8] Lin J, Cummings OW, Greenson JK et al. IgG4-related sclerosing cholangitis in the absence of autoimmune pancreatitis mimicking extrahepatic cholangiocarcinoma. Scand J Gastroenterol 2015; 50: 447 453

[9] Zaydfudim VM, Wang AY, de Lange EE et al. IgG4-associated cholangitis can mimic hilar cholangiocarcinoma. Gut Liver 2015; 9: 556 560

[10] Kamisawa T, Takuma K, Anjiki H et al. Sclerosing cholangitis associated with autoimmune pancreatitis differs from primary sclerosing cholangitis. World J Gastroenterol 2009; 15: 2357 - 2360

[11] Nakazawa T, Ohara H, Sano $\mathrm{H}$ et al. Schematic classification of sclerosing cholangitis with autoimmune pancreatitis by cholangiography. Pancreas 2006; 32: 229

[12] Nakazawa T, Ohara H, Sano $\mathrm{H}$ et al. Cholangiography can discriminate sclerosing cholangitis with autoimmune pancreatitis from primary sclerosing cholangitis. Gastrointest Endosc 2004; 60: 937 - 944

[13] Hirano K, Tada M, Isayama H et al. Endoscopic evaluation of factors contributing to intrapancreatic biliary stricture in autoimmune pancreatitis. Gastrointest Endosc 2010; 71: 85-90

[14] Inui K, Yoshino J, Miyoshi H. Differential diagnosis and treatment of biliary strictures. Clin Gastroenterol Hepatol 2009; 7: S79-83

[15] Naitoh I, Nakazawa T, Hayashi K et al. Comparison of intraductal ultrasonography findings between primary sclerosing cholangitis and IgG4-related sclerosing cholangitis. J Gastroenterol Hepatol 2015; 30: $1104-1109$

[16] Naitoh I, Nakazawa T, Ohara H et al. Endoscopic transpapillary intraductal ultrasonography and biopsy in the diagnosis of IgG4-related sclerosing cholangitis. J Gastroenterol 2009; 44: 1147-1155

[17] Kawakami H, Zen Y, Kuwatani M et al. IgG4-related sclerosing cholangitis and autoimmune pancreatitis: histological assessment of biopsies from Vater's ampulla and the bile duct. J Gastroenterol Hepatol 2010; 25: $1648-1655$

[18] Huggett MT, Culver EL, Kumar M et al. Type 1 autoimmune pancreatitis and IgG4-related sclerosing cholangitis is associated with extrapancreatic organ failure, malignancy, and mortality in a prospective UK cohort. Am J Gastroenterol 2014; 109: 1675-1683 
[19] Tanaka A, Tazuma S, Okazaki K et al. Clinical features, response to treatment, and outcomes of IgG4-related sclerosing cholangitis. Clin Gastroenterol Hepatol 2017; 15: 920 - 926 e923

[20] Hart PA, Kamisawa T, Brugge WR et al. Long-term outcomes of autoimmune pancreatitis: a multicentre, international analysis. Gut 2013; 62: $1771-1776$

[21] Andriulli A, Loperfido S, Napolitano G et al. Incidence rates of postERCP complications: a systematic survey of prospective studies. Am J Gastroenterol 2007; 102: 1781-1788

[22] Barkay O, Khashab M, Al-Haddad M et al. Minimizing complications in pancreaticobiliary endoscopy. Curr Gastroenterol Rep 2009; 11: $134-141$

[23] Ismail S, Kylanpaa L, Mustonen $\mathrm{H}$ et al. Risk factors for complications of ERCP in primary sclerosing cholangitis. Endoscopy 2012; 44: $1133-1138$

[24] Kapral C, Muhlberger A, Wewalka F et al. Quality assessment of endoscopic retrograde cholangiopancreatography: results of a running nationwide Austrian benchmarking project after 5 years of implementation. Eur J Gastroenterol Hepatol 2012; 24: 1447-1454

[25] Loperfido S, Angelini G, Benedetti G et al. Major early complications from diagnostic and therapeutic ERCP: a prospective multicenter study. Gastrointest Endosc 1998; 48: 1 - 10

[26] Masci E, Toti G, Mariani A et al. Complications of diagnostic and therapeutic ERCP: a prospective multicenter study. Am J Gastroenterol 2001; 96: 417-423
[27] Chandrasekhara V, Khashab MA, Muthusamy VR et al. Adverse events associated with ERCP. Gastrointest Endosc 2017; 85: 32-47

[28] Freeman ML, Nelson DB, Sherman S et al. Complications of endoscopic biliary sphincterotomy. NEJM 1996; 335: 909-918

[29] Bi Y, Hart PA, Law R et al. Obstructive jaundice in autoimmune pancreatitis can be safely treated with corticosteroids alone without biliary stenting. Pancreatology 2016; 16: 391-396

[30] Kamisawa T, Okazaki K, Kawa S et al. Amendment of the Japanese Consensus Guidelines for Autoimmune Pancreatitis, 2013 III. Treatment and prognosis of autoimmune pancreatitis. J Gastroenterol 2014; 49: $961-970$

[31] Cotton PB, Lehman G, Vennes J et al. Endoscopic sphincterotomy complications and their management: an attempt at consensus. Gastrointest Endosc 1991; 37: 383-393

[32] Isayama H, Hamada T, Yasuda I et al. TOKYO criteria 2014 for transpapillary biliary stenting. Dig Endosc 2015; 27: 259-264

[33] Iwasaki S, Kamisawa T, Koizumi S et al. Assessment in steroid trial for IgG4-related sclerosing cholangitis. Adv Med Sci 2015; 60: 211-215

[34] Naitoh I, Nakazawa T, Okumura F et al. Endoscopic retrograde cholangiopancreatography-related adverse events in patients with type 1 autoimmune pancreatitis. Pancreatology 2016; 16: $78-82$

[35] Moon SH, Kim MH, Park DH et al. Is a 2-week steroid trial after initial negative investigation for malignancy useful in differentiating autoimmune pancreatitis from pancreatic cancer? A prospective outcome study Gut 2008; 57: $1704-1712$ 